\title{
The bioweapons convention's impact on bioindustry
}

\section{Richard Sullivan and Sebastian Gorka}

Despite being signed in 1972, the Biological and Toxins Weapons Convention (BTWC) still awaits rigorous verification and enforcement across the globe. The success of counterproliferation and control regimes currently under development by the United Nations' Ad Hoc Group on Biological Weapons will depend on participation and cooperation of the biopharmaceutical industry.

Commercial biotechnology has often served as a front for governments involved in covert research on biological weapons. Companies allow political elites to distance themselves from illegal research programs that otherwise would lead to swift and tough censure by the international community. Today, US intelligence estimates conclude that some 24 countries possess biological weapons or have active programs to develop them. The urgent need for a BTWC verification regime to ensure effective global surveillance of bioweapon research has resulted in the suggestion by the BTWC Ad Hoc Group of a threetier approach that would include field investigations, facility investigations (to monitor activities prohibited under Article I of the BTWC), and the investigation of illegal transfers (defined under Article III of the BTWC).

Although EuropaBio (Brussels) and the Association of Pharmaceutical Research and Manufacturers of America (Washington, DC) have recognized the need for surveillance, they have condemned plans by the Working Group to introduce routine inspection and off-site analysis, both because confidential business information (CBI) might be compromised and because there is already a substantial regulatory burden placed on companies by other national bodies (e.g., to monitor good manufacturing practice). In addition, the cost, technical considerations, and current immaturity of inspection technology suggest that such industry inspections may not be a realistic option.

In the event that a particular company or factory is identified by the various intelligence services as potentially breaching articles of the BTWC, there are probably more than adequate

Richard Sullivan is clinical research fellow, Physiology Department, University College London, and head of clinical programs for the Cancer Research Campaign of the UK. Sebastian Gorka is director, US Council for Emerging National Security Affairs, previously of the RAND Corporation's National Security Research Division, Washington DC (Gorka@compuserve.com). legal processes already in place to allow a challenge visit. What the strengthened BTWC would have at its disposal is the twin pillars of verification: namely routine and challenge visits.

Routine visits are likely to be enforced by the international Organization for the Prevention of Biological Warfare (OPBW), which will probably adopt as its operational mandate some of the 21 verification measures reported by the Ad Hoc Group under the anodyne title of the "Ad Hoc Group of Government Experts to Identify and Examine Verification Measures from a Scientific and Technical Standpoint" (VEREX). These can effectively be distilled down to two measures: site surveillance and export surveillance.

Despite the fact that the United Nations' Special Commission (UNSCOM) reported that largely unremarkable materials and agents were used to build Iraq's biological weapon program, a great deal of pressure has been brought to bear on industry and governments to devise rigorous systems for detecting and tracking potential biological warfare program buildups. Unlike nuclear and (certain) chemical weapons, the technology required to produce biological warfare is nearly always dual use.

The response by policymakers has been to formulate "signature lists" of potential agents and equipment that may flag or indicate a potential biological warfare threat. The former has proved relatively easier to identify and enumerate, although this may be impractical with the increasing use of genetic engineering. Equipment assessments have identified certain fermentation, separation, and biohazard containment items as being potential signature equipment. In theory, interrogation of an integrated global logistical database of sales from industry should flag potential sales of restricted items or demonstrate volumes (e.g., growth media) incompatible with the requirements for academic research.

Unfortunately, this approach has two problems. First, it would require costly and intrusive surveillance into confidential industry databases, not to mention the massive problems associated with collating information from different systems. Second, it assumes that the type of signature equipment that a nation-state, terrorist group, or individual would require to develop biological weapons can be predicted with reasonable accuracy on the basis of the US/UK's own research into biological warfare. However, UNSCOM's experience with Iraq demonstrated that scant attention was paid to biosafety during weapon production, indicating there was little such signature equipment (e.g., double-ended sterilizers, air-fed suits).

One solution would be for companies to supply raw data that would then be collated (globally) and analyzed by an intelligence agency (or agencies). In such a scheme, both practical and legislative measures would be required to ensure that industry $\mathrm{CBI}$ was not transferred to other intelligence departments within governments for the purpose of economic espionage. Another solution is for an industry-centered vigilance and reporting system for potential dual-use exports and restricted biological agents.

Until recently, the US was the largest exporter of dual-use technology, restricted microorganisms, and toxins. In 1994 alone, some 531 export licenses were issued for the last group (4 were denied). The majority of exports from industry actually have no direct link to biological warfare export controls, and as such the hyperbole surrounding increased restrictions on industry exports damaging trade is rather unjustified. There is no evidence that present controls or any future additional controls will stifle or damage peaceful international trade, as long as they are applied across the global market.

There can be little doubt that the BWTC needs strengthening, not least as an act of reinforcement for the message that bioweapons are totally unacceptable. What the UN working groups have been attempting to create is both an international environment conducive to cooperation and pragmatic solutions to the inevitably tangled protocols of verification, export control, and so on. The issue for the biopharmaceutical industry is whether it should actively participate in the process.

We believe the answer should be yes. Industry must be proactive in the development of new counterproliferation and control regimes to ensure that industry self-regulatory and declarations mechanisms negate the need for far-reaching and potentially damaging nonchallenge visits and off-site testing. Part of this self-regulation should incorporate safeguards against unauthorized access and/or transfer of dual-use agents and technology to high-risk states and personnel. In this regard, lessons can be learned from the chemical industry, which successfully developed and implemented "managed access," procedures for the chemical weapons convention in conjunction with chemical industry officials and with due regard for CBI. This will undoubtedly represent the way forward for the biopharmaceutical industry. I// 